TRANSACTIONS OF THE

AMERICAN MATHEMATICAL SOCIETY

Volume 354, Number 4, Pages 1699-1703

S 0002-9947(01)02938-5

Article electronically published on October 26, 2001

\title{
AN ESTIMATE FOR WEIGHTED HILBERT TRANSFORM VIA SQUARE FUNCTIONS
}

\author{
S. PETERMICHL AND S. POTT
}

\begin{abstract}
We show that the norm of the Hilbert transform as an operator on the weighted space $L^{2}(w)$ is bounded by a constant multiple of the $3 / 2$ power of the $A_{2}$ constant of $w$, in other words by $c \sup _{I}\left(\langle\omega\rangle_{I}\left\langle\omega^{-1}\right\rangle_{I}\right)^{3 / 2}$. We also give a short proof for sharp upper and lower bounds for the dyadic square function.
\end{abstract}

\section{INTRODUCTION}

The question of finding sharp estimates for the Hilbert transform, the square function and a uniform bound for martingales on weighted $L^{2}$ spaces in terms of the $A_{2}$ constant of the weight has attracted considerable interest in recent years. S. Buckley proved in [1] that the norm of the square function is bounded by $Q_{2}(\omega)^{3 / 2}$ and that the Hilbert transform is bounded by $Q_{2}(\omega)^{2}$. More recently, S. Hukovic, $\mathrm{S}$. Treil and A. Volberg proved in [3] the linear bound for the square function. An alternative proof by J. Wittwer can be found in [7].

We improve Buckley's bound for the Hilbert transform to $Q_{2}(\omega)^{3 / 2}$. Our proof uses a certain averaging technique introduced by the first author in [5]. The new bound for the Hilbert transform follows from upper and lower bounds for the square function in just one line.

The authors are grateful to A. Volberg and F. Nazarov for valuable discussions.

\section{Formulation of Result}

We consider the space $L_{\mathbb{R}}^{2}(\omega)$ where $\omega$ is a positive $L_{l o c}^{1}$ function, called a weight. Let $d x$ be Lebesgue measure on $\mathbb{R}$. The norm of $f \in L_{\mathbb{R}}^{2}(\omega)$ is $\left(\int_{\mathbb{R}}|f(x)|^{2} \omega(x) d x\right)^{1 / 2}$ and denoted by $\|f\|_{\omega}$. We are concerned with a special class of weights, called $A_{2}$. We say that $\omega \in A_{2}$, if

$$
Q_{2}(\omega):=\sup _{I}\langle\omega\rangle_{I}\left\langle\omega^{-1}\right\rangle_{I}<\infty
$$

where the supremum is taken over all intervals $I \subset \mathbb{R}$. The notation $\langle\omega\rangle_{I}$ stands for the average of the function $\omega$ over $I$.

Let $\mathcal{D}$ denote the collection of all dyadic intervals in $\mathbb{R}$. We call $\mathcal{D}$ the standard dyadic grid in $\mathbb{R}$. For each $\alpha \in \mathbb{R}, r>0$, let $\mathcal{D}^{\alpha, r}$ be the dyadic grid $\{\alpha+r I: I \in \mathcal{D}\}$.

Received by the editors August 15, 2001.

1991 Mathematics Subject Classification. Primary 42A50; Secondary 42A61.

Key words and phrases. Weighted norm inequalities, square function, Hilbert transform.

The second author gratefully acknowledges support by EPSRC and thanks the Mathematics Department at MSU for its hospitality. 
If we restrict the supremum in (2.1) to dyadic intervals of a certain grid, we will denote the class by $A_{2}^{\mathcal{D}^{\alpha, r}}$ and the corresponding supremum by $Q_{2}^{\mathcal{D}^{\alpha, r}}(\omega)$.

The symbol $H$ stands for the Hilbert transform on $\mathbb{R}$, which is defined as

$$
H f(x)=p . v \cdot \int_{\mathbb{R}} \frac{f(y)}{x-y} d y .
$$

Here is the main result of this paper:

Theorem 2.1. $H: L_{\mathbb{R}}^{2}(\omega) \rightarrow L_{\mathbb{R}}^{2}(\omega)$ has operator norm $\|H\| \leq c Q_{2}(\omega)^{3 / 2}$, where $c>0$ is an absolute constant.

We will reduce the problem to upper and lower bounds of certain square functions, using the averaging technique from $[\mathbf{5}$.

\section{SHARP LOWER AND UPPER BOUNDS FOR THE DYADIC SQUARE FUNCTION}

The following considerations hold for all dyadic grids $\mathcal{D}$, so we omit indices $\alpha, r$. Recall that the dyadic square function $S$ is defined by

$$
S f(t)=\sqrt{\int_{\Sigma}\left|\left(T_{\varepsilon} f\right)(t)\right|^{2} d \varepsilon}=\sqrt{\sum_{I \in \mathcal{D}}\left|\left(f, h_{I}\right)\right|^{2} \frac{\chi_{I}(t)}{|I|}},
$$

where $\Sigma$ denotes the space $\{-1,1\}^{\mathcal{D}}$ equipped with the natural product measure $d \varepsilon$, which assigns equal measure $2^{-k}$ to every cylindrical subset of $\{-1,1\}^{\mathcal{D}}$ of length $2^{k} . T_{\varepsilon}$ is the martingale transform $f=\sum_{I}\left(f, h_{I}\right) h_{I} \mapsto \sum_{I} \varepsilon(I)\left(f, h_{I}\right) h_{I}$ associated to the sequence $\varepsilon(I) \in\{-1,1\}^{\mathcal{D}}$.

We first prove a lower bound for the square function.

Theorem 3.1. There exists $c>0$ so that for all $f \in L^{2}(\omega)$,

$$
\|f\|_{\omega} \leq c Q_{2}^{\mathcal{D}}(\omega)^{1 / 2}\|S f\|_{\omega} .
$$

Proof. We have

$$
\|S f\|_{\omega}^{2}=\sum_{I}\langle\omega\rangle_{I}\left|\left(f, h_{I}\right)\right|^{2}=\left(D_{\omega} f, f\right),
$$

where $D_{\omega}$ stands for 'discrete multiplication' by $\omega$ and denotes the possibly unbounded operator which is densely defined on $L^{2}$ by $h_{I} \mapsto\langle\omega\rangle_{I} h_{I}$. Let $M_{\omega}$ denote the ordinary multiplication operator with $\omega$. Of course, $\|f\|_{\omega}^{2}=\left(M_{\omega} f, f\right)$. We need to show that

$$
M_{\omega} \leq c Q_{2}^{\mathcal{D}}(\omega) D_{\omega} .
$$

Here, the inequality is understood as an operator inequality.

Approximating $\omega$ by $\omega_{n}$, where $\omega_{n}(x)=\max \{\min \{\omega(x), n\}, 1 / n\}$, we can assume that $M_{\omega}$ and $D_{\omega}$ are bounded and invertible. Taking inverses, equation (3.1) becomes

$$
D_{\omega}^{-1} \leq c Q_{2}^{\mathcal{D}}(\omega) M_{\omega}^{-1}
$$

where $D_{\omega}^{-1}$ is defined by $h_{I} \mapsto\langle\omega\rangle_{I}^{-1} h_{I}$, and $M_{\omega}^{-1}=M_{\omega^{-1}}$. So we need to prove that

$$
\sum_{I} \frac{1}{\langle\omega\rangle_{I}}\left|\left(f, h_{I}\right)\right|^{2} \leq c Q_{2}^{\mathcal{D}}(\omega)\|f\|_{\omega^{-1}}^{2}
$$


We switch to the system of disbalanced Haar functions $h_{I}^{\omega}$ that is orthonormal in $L_{\omega}^{2}$, as done in [3]. For this, we define $h_{I}^{\omega}$ as $h_{I}=\delta_{\omega}^{I} h_{I}^{\omega}+\gamma_{\omega}^{I} \chi_{I}$, where

$$
\delta_{\omega}^{I}=\sqrt{\frac{\langle\omega\rangle_{I_{+}}\langle\omega\rangle_{I_{-}}}{\langle\omega\rangle_{I}}} \text { and } \gamma_{\omega}^{I}=\frac{\left(\omega, h_{I}\right)}{|I|\langle\omega\rangle_{I}} .
$$

Furthermore, we write $\Delta_{I} \omega$ for $\langle\omega\rangle_{I_{-}}-\langle\omega\rangle_{I_{+}}=|I|^{-1 / 2}\left(\omega, h_{I}\right)$.

We now split the sum into three parts:

$$
\begin{aligned}
\sum_{I} \frac{1}{\langle\omega\rangle_{I}}\left|\left(f, h_{I}\right)\right|^{2} & =\sum_{I} \frac{1}{\langle\omega\rangle_{I}}\left|\delta_{\omega}^{I}\right|^{2}\left|\left(f, h_{I}^{\omega}\right)\right|^{2} \\
& +2 \sum_{I} \frac{1}{\langle\omega\rangle_{I}}\left|\delta_{\omega}^{I}\right|\left|\gamma_{\omega}^{I}\right|\left|\left(f, h_{I}^{\omega}\right)\right|\left|\left(f, \chi_{I}\right)\right|+\sum_{I} \frac{1}{\langle\omega\rangle_{I}}\left|\gamma_{\omega}^{I}\right|^{2}\left|\left(f, \chi_{I}\right)\right|^{2} .
\end{aligned}
$$

The first sum. Note that $\frac{\left(\delta_{\omega}^{I}\right)^{2}}{\langle\omega\rangle_{I}} \leq 1$, so

$$
\begin{aligned}
\sum_{I} \frac{1}{\langle\omega\rangle_{I}}\left|\delta_{\omega}^{I}\right|^{2}\left|\left(f, h_{I}^{\omega}\right)\right|^{2} & \leq \sum_{I}\left|\left(f, h_{I}^{\omega}\right)\right|^{2}=\sum_{I}\left|\left(\omega^{-1} f, h_{I}^{\omega}\right)_{\omega}\right|^{2} \\
& =\left\|\omega^{-1} f\right\|_{\omega}^{2}=\|f\|_{\omega^{-1}}^{2}
\end{aligned}
$$

The second sum.

$$
\begin{aligned}
& \sum_{I} \frac{1}{\langle\omega\rangle_{I}}\left|\delta_{\omega}^{I}\left\|\gamma_{\omega}^{I}\right\|\left(f, h_{I}^{\omega}\right)\right|\left|\left(f, \chi_{I}\right)\right| \\
& \quad \leq \sqrt{\sum_{I} \frac{1}{\langle\omega\rangle_{I}}\left(\delta_{\omega}^{I}\right)^{2}\left|\left(f, h_{I}^{\omega}\right)\right|^{2}} \sqrt{\sum_{I} \frac{1}{\langle\omega\rangle_{I}}\left(\gamma_{\omega}^{I}\right)^{2}\left|\left(f, \chi_{I}\right)\right|^{2}}
\end{aligned}
$$

where the first part can be estimated by $\|f\|_{\omega^{-1}}$ as above. The second term is exactly the square root of the third sum and will be estimated below.

\section{The third sum.}

$$
\sum_{I} \frac{1}{\langle\omega\rangle_{I}}\left|\gamma_{\omega}^{I}\right|^{2}\left|\left(f, \chi_{I}\right)\right|^{2}=\sum_{I}|I| \frac{\left|\Delta_{I} \omega\right|^{2}}{\langle\omega\rangle_{I}^{3}}\langle f\rangle_{I}^{2}
$$

We will apply the weighted Carleson Imbedding theorem to control (3.6). According to [4], it suffices to check (3.6) for test functions, in the sense that any sequence $\alpha_{I} \geq 0$ satisfying

$$
\frac{1}{|J|} \sum_{I \subset J}\langle\omega\rangle_{I}^{2} \alpha_{I} \leq C\langle\omega\rangle_{J} \text { for all dyadic } J
$$

also satisfies

$$
\sum_{I}\langle f\rangle_{I}^{2} \alpha_{I} \leq 4 C\|f\|_{\omega^{-1}}^{2}
$$

for all $f \in L^{2}\left(w^{-1}\right)$.

We apply this to $\alpha_{I}=|I| \frac{\left|\Delta_{I} \omega\right|^{2}}{\langle\omega\rangle_{I}^{3}}$, and $C=c Q_{2}^{\mathcal{D}}$. So it suffices to check that for all dyadic $J$,

$$
\frac{1}{|J|} \sum_{I \subset J}|I| \frac{\left|\Delta_{I} \omega\right|^{2}}{\langle\omega\rangle_{I}} \leq c Q_{2}^{\mathcal{D}}\langle\omega\rangle_{J} .
$$


This has been proven in [7] and can also be shown by a Bellman function argument.

Corollary 3.2. There exists $c>0$ such that for all $f \in L^{2}(\omega)$ and for all weights $\omega,\|S f\|_{\omega} \leq c Q_{2}^{\mathcal{D}}(\omega)\|f\|_{\omega}$.

Proof. Using the same notation as before, we have to show that $D_{\omega} \leq c Q_{2}^{\mathcal{D}}(\omega)^{2} M_{\omega}$. By definiton of $Q_{2}^{\mathcal{D}}(\omega)$, we have $D_{\omega} \leq Q_{2}^{\mathcal{D}}(\omega)\left(D_{\omega^{-1}}\right)^{-1}$, and by equation (3.2) applied to $\omega^{-1}$ we obtain $D_{\omega} \leq c Q_{2}^{\mathcal{D}}(\omega)^{2} M_{\omega}$.

Remark. This corollary was proven in [3] using Bellman function technique. The paper [7] also contains a short proof of the fact that the lower bound in Theorem 3.1 implies the linear upper bound for $S$ of Theorem 3.2, which itself is sharp (see [1] and [3]). In particular, this argument shows that the lower bound in Theorem 3.1 is sharp.

\section{The Cubic Bound for the Hilbert transform}

By [5], $H$ lies in the closed convex hull of operators densely defined by

$$
\amalg^{\alpha, r} h_{I}=\frac{1}{\sqrt{2}}\left(h_{I_{-}}-h_{I_{+}}\right) .
$$

We will refer to these operators as dyadic shifts. The indices $\alpha$ and $r$ indicate that we have to consider translates and dilates of the standard dyadic grid as described above. The square function does not 'see' the dyadic shift:

Proposition 4.1. $(S \amalg f)(x)=(S f)(x)$ for all $x$.

Proof.

$$
\begin{aligned}
S \amalg f(x)^{2} & =\int_{\Sigma}\left|\left(T_{\varepsilon} \amalg f\right)(x)\right|^{2} d \varepsilon=\int_{\Sigma}\left|\sum_{I} \varepsilon(I)\left(\amalg f, h_{I}\right) h_{I}(x)\right|^{2} d \varepsilon \\
& =\int_{\Sigma}\left|\sum_{I}\left(f, h_{I}\right)\left(\varepsilon\left(I_{-}\right) h_{I_{-}}-\varepsilon\left(I_{+}\right) h_{I_{+}}\right)\right|^{2} d \varepsilon \\
& \stackrel{(\star)}{=} \int_{\Sigma}\left|\sum_{I} \varepsilon(I)\left(f, h_{I}\right) h_{I}(x)\right|^{2} d \varepsilon=S f(x)^{2} .
\end{aligned}
$$

Here, $(\star)$ is an effect of the averaging over sequences of signs $\varepsilon(I)$ and the fact that for each fixed $x$ there exists a sequence of signs $\tilde{\varepsilon}(I)$ so that $\sqrt{2} h_{I}(x)=$ $\tilde{\varepsilon}(I)\left(\varepsilon\left(I_{-}\right) h_{I_{-}}-\varepsilon\left(I_{+}\right) h_{I_{+}}\right)(x)$.

Now it is easy to prove Theorem 2.1:

Proof. Dyadic shifts with respect to all translates and dilates of the standard dyadic grid have cubic bound, indeed,

$$
\begin{aligned}
\left\|\amalg^{\alpha, r} f\right\|_{\omega} & \stackrel{(1)}{\leq} c Q_{2}^{\mathcal{D}^{\alpha, r}}(\omega)^{1 / 2}\left\|S \amalg^{\alpha, r} f\right\|_{\omega} \stackrel{(2)}{=} c Q_{2}^{\mathcal{D}^{\alpha, r}}(\omega)^{1 / 2}\|S f\|_{\omega} \\
& \stackrel{(3)}{\leq} c Q_{2}^{\mathcal{D}^{\alpha, r}}(\omega)^{3 / 2}\|f\|_{\omega},
\end{aligned}
$$

where (1) holds by Theorem 3.1 (2) by Proposition 4.1 and (3) by Corollary 3.2 
By convexity, we now obtain the desired bound for the Hilbert transform:

$$
\|H\|_{L^{2}(\omega) \rightarrow L^{2}(\omega)} \leq c \sup _{\alpha, r}\left\|\amalg^{\alpha, r}\right\|_{L^{2}(\omega) \rightarrow L^{2}(\omega)} \leq c \sup _{\alpha, r} Q_{2}^{\mathcal{D}^{\alpha, r}}(\omega)^{3 / 2} \leq c Q_{2}(\omega)^{3 / 2} .
$$

This finishes the proof of the main result.

Remark. After this paper was submitted, the first author improved the bound to $Q_{2}(\omega)$, which is sharp [6]. However, the proof is much more involved than the proof of the $Q_{2}(\omega)^{3 / 2}$ bound, which we present in this paper.

\section{REFERENCES}

[1] S. M. BuCKLeY, Summation Condition on Weights, Michigan Math. J., 40(1), pp. 153-170, 1993. MR 94d:42021

[2] S. Hukovic, Thesis, Brown University, 1998.

[3] S. Hukovic, S. Treil, A. Volberg, The Bellman Functions and Sharp Weighted Inequalities for Square Functions, Operator Theory: Advances and Applications, v.113, Birkhäuser Verlag, 2000. MR 2001j:42012

[4] F. Nazarov, S. Treil, A. Volberg, The Bellman functions and two weight inequalities for Haar multipliers, J. Amer. Math. Soc, v.12, no. 4, pp. 909-928, 1999. MR 2000k:42009

[5] S. Petermichl Dyadic shifts and a logarithmic estimate for Hankel operators with matrix symbol, Comptes Rendus Acad. Sci. Paris, t.330, no.6, pp. 455-460, 2000.

[6] S. Petermichl A sharp estimate of the weighted Hilbert transform via classical $A_{p}$ characteristic, Preprint, Insitute of Advanced Studies, 2001.

[7] J. WitTwer, A sharp estimate on the norm of the martingale transform, Math. Res. Lett., v.7, pp. 1-12, 2000. MR 2001e:42022

Department of Mathematics, Michigan State University, East Lansing, Michigan 48824-1027

Current address: Institute of Advanced Studies, Princeton, New Jersey 08540

E-mail address: stefanie@math.msu.edu

Department of Mathematics, University of York, York YO10 5DD, UK

E-mail address: sp23@york.ac.uk 